Koçi, R. [et al.]. PatternLens: Inferring evolutive patterns from web API usage logs. A: International Conference on Advanced Information Systems Engineering. "Intelligent Information Systems, CAiSE Forum 2021: Melbourne, VIC, Australia, June 28-July 2, 2021: proceedings". Berlín: Springer, 2021, p. 146-153. ISBN 978-3-030-79108-7.

The final authenticated version is available online at https://doi.org/10.1007/978-3-030-79108-7_17

\title{
PatternLens: Inferring evolutive patterns from web API usage logs
}

\author{
Rediana Koçi, Xavier Franch, Petar Jovanovic, Alberto Abelló \\ Universitat Politècnica de Catalunya, BarcelonaTech \\ $\{$ koci, franch, petar, aabello\}@essi.upc.edu
}

\begin{abstract}
The use of web Application Programming Interfaces (WAPIs) has experienced a boost in recent years. Developers (i.e., WAPI consumers) are continuously relying on third-party WAPIs to incorporate certain features into their applications. Consequently, WAPI evolution becomes more challenging in terms of the service provided according to consumers' needs. When deciding on which changes to perform, besides several dynamic business requirements (from the organization whose data are exposed), WAPI providers should take into account the way consumers use the WAPI. While consumers may report various bugs or may request new endpoints, their feedback may be partial and biased (based on the specific endpoints they use). Alternatively, WAPI providers could exploit the interaction between consumers and WAPIs, which is recorded in the WAPI usage logs, generated while consumers access the WAPI. In this direction, this paper presents PatternLens, a tool with the aim of supporting providers in planning the changes by analyzing WAPI usage logs. With the use of process mining techniques, this tool infers from the logs a set of usage patterns (e.g., endpoints that are frequently called one after the other), whose occurrences imply the need for potential changes (e.g., merging the two endpoints). The WAPI providers can accept or reject the suggested patterns, which will be displayed together with informative metrics. These metrics will help providers in the decision-making, by giving them information about the consequences of accepting/rejecting the suggestions.
\end{abstract}

Keywords: Web API evolution, pattern detection, process mining, web API change

\section{Introduction}

As the use of web Application Programming Interfaces (WAPIs) is increasingly growing, their evolution becomes more challenging in terms of the service provided according to consumers' needs. While a lot of effort is put in analyzing consumers' struggles when WAPIs change $[4,5,7]$, little is known about providers' burdens: what, how, and when to evolve [1]. When deciding on which changes to perform, besides several business requirements (from the organization whose data are exposed), providers should take into account the way consumers use 
the WAPI. Moreover, knowing the impact that changes usually cause to the consumers, they have to strike a balance between not imposing irrelevant, unexpected, frequent changes and providing an up-to-date, maintainable, bug-free WAPI, that fulfills their needs [3].

While consumers may report various bugs or may request new endpoints (i.e., URLs to access WAPIs resources), their feedback may be partial and biased (based on the specific endpoints they use), as well as difficult to gather and interpret at scale [9]. Alternatively, WAPI providers could exploit the interaction between consumers and WAPIs, which is recorded in the WAPI usage logs, generated while consumers access the WAPI. Every time a consumer application makes a request, a log entry is generated and stored in the usage log file. Therefore, consumers' behavior is recorded in these logs, and their analysis can obliquely reveal consumers' needs for new features hidden under several workarounds (solutions found by WAPI users that allow them to get data, functionality, or features they need, but that are not yet implemented by providers), or find room for potential improvements.

As applications are the actual WAPI consumers, we should consider the different ways they consume WAPIs over their own lifecycle. Basically, applications interact with the WAPIs during design time and runtime, over both of which they manifest different aspects of their behavior. Following on from this, we distinguish two types of logs: (i) development logs, generated while developers build and test their applications and (ii) production logs, generated while applications are being used by end users, meaning that the WAPI requests are predetermined by the implemented functionalities of the applications. We make this distinction as each of these log types, even though provide useful information about WAPI consumption, can be analyzed in different ways. But how much are these logs used and analyzed, beyond the typical traffic monitoring? Several works $[8,2]$ suggest analyzing development logs to measure the usability of WAPIs and thus perform changes that increase the perceived usability from consumers point of view. In addition, there are various WAPI monitoring tools available that take as input the WAPI usage logs, but they are mostly oriented toward providing reporting dashboards or automatic alerting in case of WAPI failure [10]. Providers have all this potentially insightful, large volume of data that is being generated, but not enough proactively used for evolution.

To address the problem of understanding consumers' needs, we present PatternLens, a tool that aims to support providers in planning the changes by analyzing consumers behavior recorded in the WAPI usage logs. We make use of process mining techniques and compute from the logs a set of metrics regarding the real consumption of WAPI endpoints by consumers, like the calling frequency of the endpoints, the frequency of a sequence of endpoints, etc. Using these metrics and a pre-defined set of patterns, we detect from the logs all the patterns, whose occurrences imply the need for potential changes (e.g., merging two endpoints called always one after the other). The WAPI providers can accept or reject the suggested patterns, which will be displayed together with informative metrics, helping providers in the decision-making. 


\section{Background: Process Mining in the WAPI context}

Process mining is a process-oriented data mining discipline that uses event logs to extract process-related knowledge. We give the definition of some fundamental concepts of process mining, as presented by Van der Aalst [6], adapt them to the WAPI domain, and use them to give the definition of concepts referred in the rest of the paper:

- Activity - a specific step in the process. For WAPIs, activities are calls to the endpoints, i.e., the WAPI resources URL.

- Case - a process instance. We refer as a case to a set of requests that an application is submitting to the WAPI during a certain time period, commonly referred as session.

- Event - an activity occurrence. An event refers to an activity and belongs to a specific case. We refer to WAPI requests as events, and identify them by activity names (request method like GET, POST, and the endpoint).

- Event $\log$ - a set of cases. A WAPI usage log contains all the requests (i.e., events) that consumers (i.e., applications) make against the WAPI.

Using these concepts, we define a sequence as an ordered occurrence of events within a case, and a pattern as a frequent sequence that when fulfilling some pre-defined conditions, indicates the need for a specific change.

In order to apply process mining, an event log should have at least three attributes: (i) case identifier that identifies the case to whom each event belongs, (ii) activity name that identifies each event, and (iii) timestamp that indicates the time when an event occurs. The presence of these three attributes allows to infer process insights from the event log. Certainly, other attributes that might store additional information about the events (e.g., device IP, application ID, status code), whenever available, add value to the analysis.

In the WAPI context, process discovery consists on creating a graph-based process model from the event log, where nodes represent the endpoints being called, and edges the calling sequence of two endpoints. Even though we do not visualize the process model, we aim at gathering statistical information about it (e.g., the frequency of calling one endpoint after another).

\section{PatternLens overview}

PatternLens takes as input the usage log file (i.e., event log) containing all the WAPI calls from several consumers' applications. We assume that the logs are already cleaned and prepared, meaning that (i) every event (i.e., request) belongs to one case (i.e., session), (ii) every event has a distinct timestamp, making able to build the right sequence of calls, and (iii) the activities' names are precise enough to identify each process step, so that no two different process steps to appear with the same activity name, and at the same time no the same process step to appear with different activity names. 
Based on process mining concepts, the tool first computes a set of metrics for each WAPI endpoint and sequence of endpoints as appearing in the file. Then, using a pre-defined set of patterns and the computed metrics, it detects and displays to the users all the patterns, of different types. Along each pattern, the users are presented with the suggested changes that the patterns imply, as well as various metrics that might help them in selecting the patterns that better fit their interest. The users are given the possibility to accept the patterns that are interesting to them (i.e., the implied changes are feasible from the providers point of view and would really improve the WAPI with regard to consumers' needs), reject the ones that do not seem interesting, or ignore the ones for whom they are neutral or do not have any conscious thought. We want to note that PatternLens is designed to be used by WAPI providers in analyzing consumers' behavior. As such, we assume that its users (i.e., providers) have a strong prior knowledge of the WAPI, needed in order to proper accept/reject patterns and the recommended changes. In the following, we introduce and explain two main parts of the tool: the metrics calculator and the patterns detector (see Figure 1).

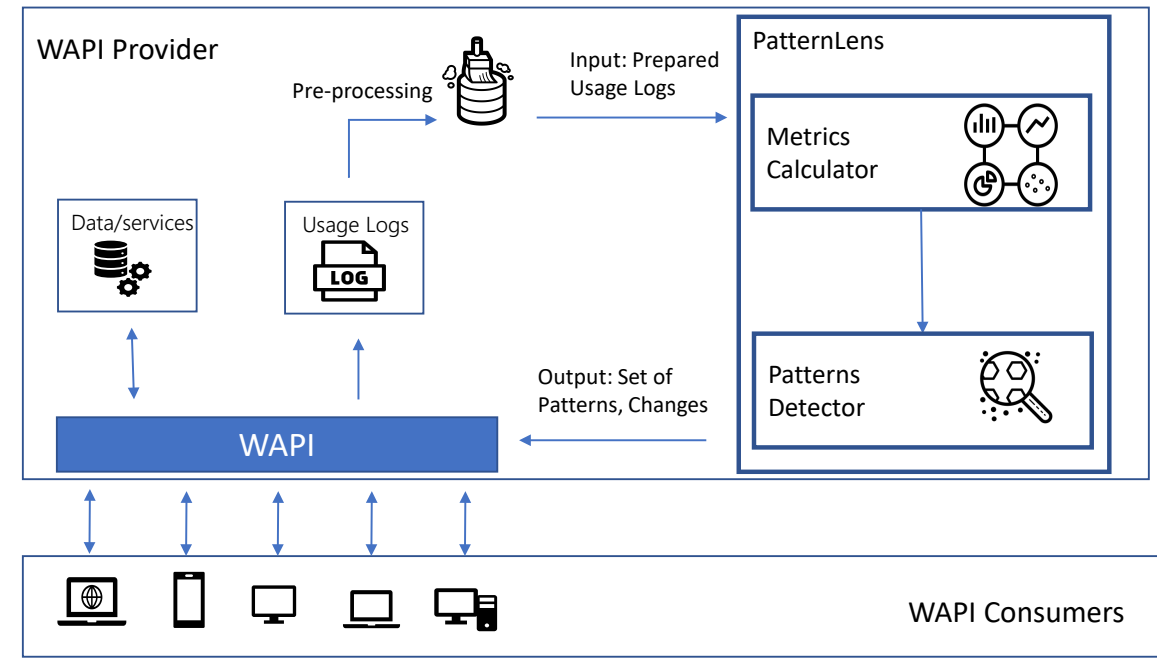

Fig. 1: PatternLens in the full context of WAPI - consumers interaction

\subsection{Metrics calculator}

PatternLens considers the interaction between consumers and WAPI as a process. As such, to build the process model, we refer to the process activities (calling method and endpoint) as nodes, and to the order two endpoints are being called by the consumers, as edges. Before detecting the patterns, PatternLens defines all the nodes and edges, by extracting for each of them a set of attributes and computing some metrics (Figure 2).

For the nodes we compute and make use of the following metrics: 


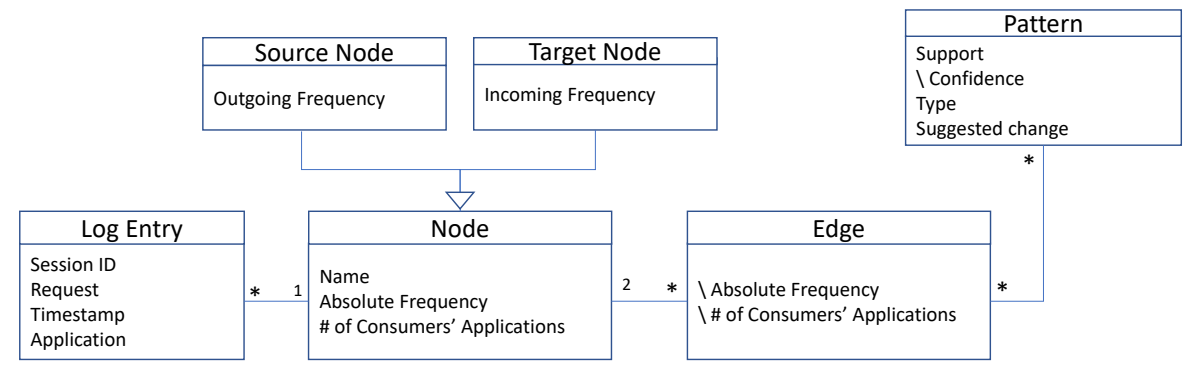

Fig. 2: PatternLens: Class Diagram

- Absolute frequency: total number of times that an endpoint was called.

- Incoming frequency (for target nodes): total number of times that an endpoint was called after other endpoints.

- Outgoing frequency (for source nodes): total number of times that an endpoint was called before other endpoints.

- \# Consumers' application: the number of applications that have called the endpoint in study.

Each edge combines two nodes: a source node (i.e., the first called endpoint in a two-node sequence), and a target node (the second called endpoint in a two-node sequence). We define the edges by the following derived metrics:

- Absolute frequency: total number of times that a sequence of endpoints was followed.

- \# Consumers' application: the number of applications that have called the sequence of endpoints in study.

\subsection{Patterns detector}

Instead of visualizing the process map with all the sequences of calls, we filter only those parts of the process, where we see a specific behavior of the way consumers use the WAPI. We pre-define the set of patterns, whose occurrence may imply the need for potential changes, rather than extracting all the frequent patterns from the model to redundantly display them to WAPI providers without giving any hints on the behavior these patterns manifest.

To measure the effectiveness of the patterns, we use two basic concepts from association rules, namely the support and confidence metrics, and adapt them in the context of our approach. We refer as support to the number of times a pattern appears in the log file. We give the users the possibility to enter the desired minimum support, based on the size of the file they upload and how specific or general they want the patterns to be. We refer as confidence to the relative frequency of the patterns, regarding the source and the target node. As such, if a pattern has a high support (i.e., high absolute frequency: it appears too 
often in the log file), but this frequency is too low comparing with the absolute frequency of the source and the target nodes, then the pattern will have a low confidence. The tool presents the patterns to the users in a tabular form as in Figure 3.

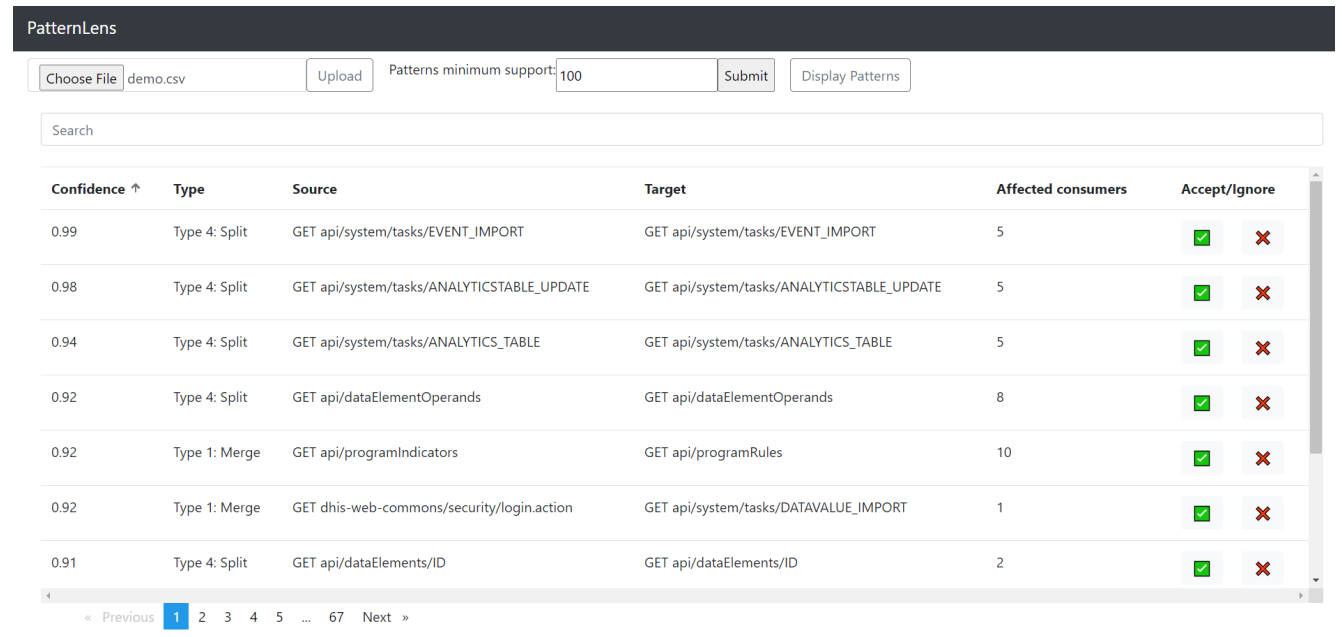

Fig. 3: PatternLens: Application Interface

We add to each pattern the metrics related to the pattern or the source/target nodes (e.g., confidence, absolute frequency, number of applications where the pattern appears), to better inform the users about the occurrence of the pattern and help them in better understanding consumers' behavior. Along to the patterns, we give a short description of the implied changes (e.g., merging the two endpoints, creating a new attribute).

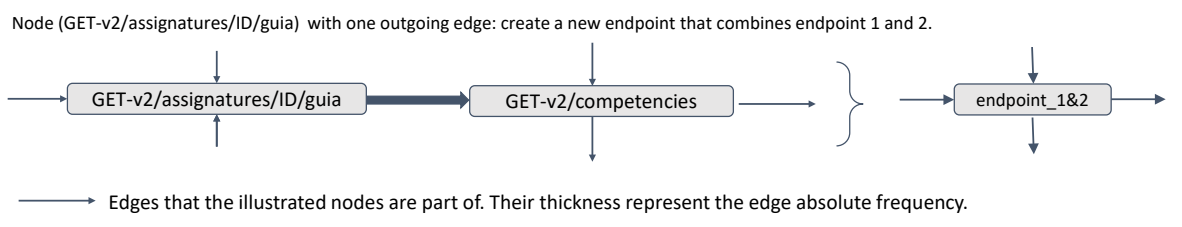

Fig. 4: The pattern and the change it implies

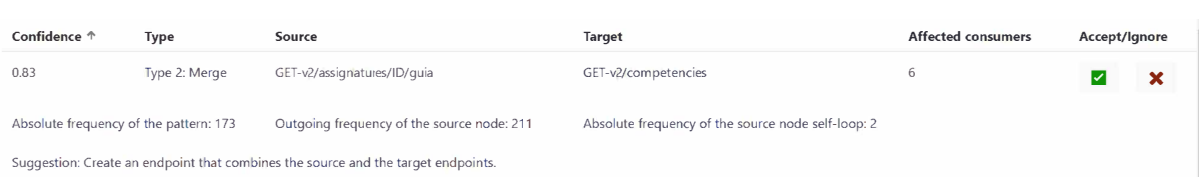

Fig. 5: The pattern as displayed in PatternLens 
Figure 4 gives an example of a pattern that the tool is able to detect. Figure 5 depicts how PatternLens displays the pattern. As seen from the Figure 4, the GET-v2/assignatures/ID/guia endpoint, responsible to get data about specific courses in university (source node), has one outgoing edge, the one toward GET$\mathrm{v} 2 /$ competencies, responsible to get data about the required competencies to take a course (target node). This means that after getting information about a specific subject, consumers always call the endpoint for the competencies of the course. For this reason, we suggest merging these two endpoints in one, that combines the data from both of them, reducing this way the number of calls consumers have to submit to get the desired data. PatternLens is able to detect this type of pattern, and displays it as in Figure 5. Along the pattern, that is defined by the source and target node, the user is informed about the confidence

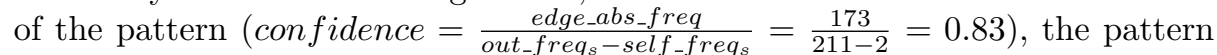
absolute frequency, the outgoing frequency of the source node, the frequency of the source node self-loop, and the change implied by the pattern. The shown metrics are different for every type of pattern, as the tool presents the most relevant ones depending on the way the pattern is being detected.

\section{Onsite demonstration}

In the onsite demonstration, we will present the functionality of PatternLens using $\log$ files from two real world examples of different domain, namely health and education. The first log file comes from the District Health Information Software 2 (DHIS2) WAPI. DHIS2 is an open source, web-based health management information system platform used worldwide from various institutions and NGOs for data entry, data quality checks and reporting. It has an open REST WAPI, used by more than 60 native applications. The second log file belongs to WAPI of the Barcelona School of Informatics at the Polytechnic University of Catalonia (Facultat d'Informàtica de Barcelona, FIB, UPC). It is built under REST architecture and is mainly being used by the FIB website, monitoring systems, school news' screen, and several applications created for academic purpose. The API provides a set of endpoints for extracting data about departments, courses, exams, rooms reservations, etc. With these two examples, we aim to show that PatternLens performs the same, despite the domain of the WAPI.

Currently, PatternLens is able to detect four different pattern types. As we assume that the users of the tool should have prior knowledge of the WAPI to decide on accepting or rejecting a pattern, and as the demo participants are not expected to be familiar with our examples, we will encourage the participants to evaluate the usability of the tool. Nevertheless, all the elements of the tool and the examples, will be well-explained, so that non WAPI experts can assess the potential of PatternLens in supporting providers in planning WAPI evolution.

\section{Future work}

We aim to further extend the PatternLens features in the following directions: 
- Provide the users with more types of patterns.

- Quantify the impact that the suggested changes may cause, if the providers decide to implement them. We plan to measure the effectiveness of the changes by predicting the excepted behavior of the consumers.

- Improve the tool according to historic data about users selections. Currently PatternLens stores information about the metrics of the patterns that users select or reject. We aim to improve the way the information is presented to them, based on their feedback.

\section{Acknowledgment}

This work is supported by GENESIS project, funded by the Spanish Ministerio de Ciencia e Innovación under project TIN2016-79269-R.

\section{References}

1. Abelló, Alberto, Claudia Ayala, Carles Farré, Cristina Gómez, Marc Oriol, and Óscar Romero. "A data-driven approach to improve the process of data-intensive API creation and evolution." In Proceedings of the 29th InternationalConference on Advanced Information Systems Engineering (CAiSE2017), pp. 1-8. CEUR-WS. org, 2017.

2. Macvean, Andrew, Luke Church, John Daughtry, and Craig Citro. "API Usability at Scale." In PPIG, 2016.

3. Koçi, Rediana, Xavier Franch, Petar Jovanovic, and Alberto Abelló. "Classification of Changes in API Evolution." In 2019 IEEE 23rd International Enterprise Distributed Object Computing Conference (EDOC), pp. 243-249. IEEE, 2019.

4. Wang, Shaohua, Iman Keivanloo, and Ying Zou. "How do developers react to restful api evolution?." In International Conference on Service-Oriented Computing, pp. 245-259. Springer, Berlin, Heidelberg, 2014.

5. Li, Jun, Yingfei Xiong, Xuanzhe Liu, and Lu Zhang. "How does web service API evolution affect clients?." In 2013 IEEE 20th International Conference on Web Services, pp. 300-307. IEEE, 2013.

6. Van Der Aalst, Wil. "Data science in action." In Process mining, pp. 3-23. Springer, Berlin, Heidelberg, 2016.

7. Espinha, Tiago, Andy Zaidman, and Hans-Gerhard Gross. "Web API growing pains: Loosely coupled yet strongly tied." Journal of Systems and Software 100 (2015): 27-43.

8. Koçi, Rediana, Xavier Franch, Petar Jovanovic, and Alberto Abelló. "A DataDriven Approach to Measure the Usability of Web APIs." In 2020 46th Euromicro Conference on Software Engineering and Advanced Applications (SEAA), pp. 6471. IEEE, 2020.

9. Zhang, Tianyi, Björn Hartmann, Miryung Kim, and Elena L. Glassman. "Enabling data-driven api design with community usage data: A need-finding study." In Proceedings of the 2020 CHI Conference on Human Factors in Computing Systems, pp. 1-13. 2020.

10. Doerrfeld, Bill. "10+ API Monitoring Tools". Available: https://nordicapis.com/10-api-monitoring-tools. 